\title{
Meteor studies in the framework of the JEM-EUSO program
}

G. Abdellaoui ${ }^{\mathrm{a}}$, S. Abe ${ }^{\mathrm{b}}$, A. Acheli ${ }^{\mathrm{c}}$, J.H. Adams J.H. ${ }^{\mathrm{d}}$, S. Ahmad ${ }^{\mathrm{e}}$, A. Ahriche ${ }^{\mathrm{f}}$, J.-N. Albert $^{\mathrm{g}}$, D. Allard ${ }^{\mathrm{h}}$, G. Alonso ${ }^{\mathrm{i}}$, L. Anchordoqui ${ }^{\mathrm{j}}, \mathrm{V}$. Andreev ${ }^{\mathrm{k}}$, A. Anzalone ${ }^{1, \mathrm{~m}}$, W. Aouimeur ${ }^{\mathrm{c}}, \mathrm{Y}$. Arai ${ }^{\mathrm{n}}$,

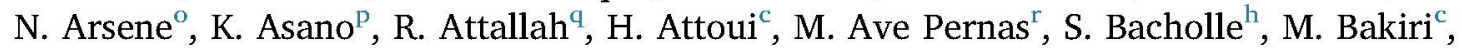
P. Baragatti ${ }^{\mathrm{s}}$, P. Barrillon ${ }^{\mathrm{g}}$, S. Bartocci ${ }^{\mathrm{s}}$, T. Batsch ${ }^{\mathrm{t}}$, J. Bayer ${ }^{\mathrm{u}}$, R. Bechini ${ }^{\mathrm{v}}$, T. Belenguer ${ }^{\mathrm{w}}$, R. Bellotti ${ }^{\mathrm{x}, \mathrm{y}}, \mathrm{A} \cdot$ Belov $^{\mathrm{z}}, \mathrm{K}$. Belov ${ }^{\mathrm{k}}$, B. Benadda ${ }^{\mathrm{a}}, \mathrm{K}$. Benmessai ${ }^{\mathrm{aa}}$, A.A. Berlind ${ }^{\mathrm{ab}}$, M. Bertaina ${ }^{\mathrm{ac}, \mathrm{v}, *}$, P.L. Biermann ${ }^{\text {ad }}$, S. Biktemerova ${ }^{\text {ae }}$, F. Bisconti ${ }^{\text {ad }}$, N. Blanc $^{\text {af }}$, J. Błęcki ${ }^{\text {ag }}$, S. Blin-Bondil ${ }^{\text {e }}$ P. Bobik ${ }^{\text {ah, }}$, M. Bogomilov ${ }^{\text {ai }}$, M. Bonamente ${ }^{\mathrm{d}}$, R. Boudaoud ${ }^{\mathrm{c}}$, E. Bozzo ${ }^{\text {aj }}$, M.S. Briggs ${ }^{\mathrm{d}}$, A. Bruno ${ }^{\mathrm{y}}$, K.S. Caballero ${ }^{\text {ak }}$, F. Cafagna ${ }^{\mathrm{x}}$, D. Campana ${ }^{\mathrm{al}}$, J.-N. Capdevielle ${ }^{\mathrm{h}}$, F. Capel $^{\mathrm{am}}$, A. Caramete ${ }^{\mathrm{o}}$, L. Caramete ${ }^{\circ}$, P. Carlson ${ }^{\text {am }}$, R. Caruso ${ }^{\text {an, } m}$, M. Casolino ${ }^{\text {ao,ap }}$, C. Cassardo ${ }^{\text {ac,v }}$, A. Castellina ${ }^{\text {ac,aq }}$, G. Castellini $^{\text {ar }}$, C. Catalano ${ }^{\text {as }}$, O. Catalano ${ }^{1, m}$, A. Cellino ${ }^{\text {ac,aq,*, M. Chikawa }}{ }^{\text {at }}$, G. Chiritoi $^{\circ}$, M.J. Christl ${ }^{\text {au }}$, V. Connaughton ${ }^{\text {, }}$, L. Conti ${ }^{\mathrm{S}, *}$, G. Cordero ${ }^{\text {av }}$, H.J. Crawford ${ }^{\text {aw }}$, R. Cremonini ${ }^{\mathrm{v}}$, S. Csorna ${ }^{\mathrm{ab}}$, S. Dagoret-Campagne ${ }^{g}$, C. De Donato ${ }^{\mathrm{ap}}$, C. de la Taille ${ }^{\mathrm{e}}$, C. De Santis ${ }^{\mathrm{ap}}$, L. del Peral ${ }^{\mathrm{r}}$, M. Di Martino aq, T. Djemil ${ }^{\mathrm{q}}$, S.A. Djenas ${ }^{\mathrm{a}}$, F. Dulucq ${ }^{\mathrm{e}}$, M. Dupieux ${ }^{\text {as }}$, I. Dutan ${ }^{\circ}$, A. Ebersoldt ${ }^{\text {ad }}$, T. Ebisuzaki ${ }^{\text {ao }}$, R. Engel ${ }^{\text {ad }}$, J. Eser ${ }^{\text {ax }}$, K. Fang ${ }^{\text {ay }}$, F. Fenu ${ }^{\text {ac,v }}$, S. Fernández-González ${ }^{\text {az }}$, J. FernándezSoriano $^{r}$, S. Ferrarese ${ }^{\text {ac,v }}$, D. Finco ${ }^{s}$, M. Flamini ${ }^{\mathrm{s}}$, C. Fornaro ${ }^{\mathrm{s}}$, M. Fouka ${ }^{\text {ba }}$, A. Franceschi ${ }^{\text {bb }}$, S. Franchini ${ }^{i}$, C. Fuglesang ${ }^{a m}$, J. Fujimoto ${ }^{\text {n }}$, M. Fukushima ${ }^{\mathrm{P}}$, P. Galeotti ${ }^{\text {ac,v }}$, E. García-Ortega ${ }^{\text {az }}$, G. Garipov ${ }^{\mathrm{z}}$, E. Gascón ${ }^{\mathrm{az}}$, J. Geary ${ }^{\mathrm{d}}$, G. Gelmini ${ }^{\mathrm{k}}$, J. Genci ${ }^{\mathrm{bc}}$, G. Giraudo ${ }^{\text {ac }}$, M. Gonchar ${ }^{\text {ac }}$, C. González Alvaradow ${ }^{\mathrm{w}}$, P. Gorodetzky ${ }^{\mathrm{h}}, \mathrm{F}$. Guarino ${ }^{\mathrm{al}, \mathrm{bd}}$, R. Guehaz ${ }^{\mathrm{c}}, \mathrm{A}$. Guzmán $^{\mathrm{u}}$, Y. Hachisu ${ }^{\text {ao }}$, M. Haiduc ${ }^{\mathrm{o}}$, B. Harlov ${ }^{\text {be }}$, A. Haungs ${ }^{\mathrm{ad}}$, J. Hernández Carretero $^{\mathrm{r}}$, W. Hidber ${ }^{\mathrm{av}}$, K. Higashide ${ }^{\mathrm{bf}, a o}$, D. Ikeda ${ }^{\mathrm{p}}$, H. Ikeda ${ }^{\mathrm{bg}}$, N. Inoue ${ }^{\mathrm{bf}}$, S. Inoue ${ }^{\mathrm{ao}}$, F. Isgrò ${ }^{\mathrm{al}, \mathrm{bh}}$, Y. Itow ${ }^{\mathrm{bi}}$, T. Jammer $^{\mathrm{bj}}$, E. Joven ${ }^{\mathrm{bk}}$, E.G. Judd ${ }^{\text {aw }}$, A. Jung ${ }^{\text {h, J. Jochum }}{ }^{\text {bj }}$, F. Kajino ${ }^{\text {bi }}$, T. Kajino ${ }^{\text {bm }}$, S. Kalli ${ }^{\text {bn }}$, I. Kaneko ${ }^{\text {ao }}$, D. Kang ${ }^{\text {ad }}$, F. Kanouni ${ }^{\text {aa }}$, Y. Karadzhov ${ }^{\text {ai }}$, J. Karczmarczyk ${ }^{\mathrm{t}}$, M. Karus ${ }^{\text {ad }}$, K. Katahira ${ }^{\text {ao }}$, K. Kawai ${ }^{\text {ao }}$, Y. Kawasaki ${ }^{\text {ao }}$, A. Kedadra ${ }^{\mathrm{c}}$, H. Khales ${ }^{\mathrm{c}}$, B.A. Khrenov ${ }^{\text {z }}$, Jeong-Sook Kim ${ }^{\text {bo }}$, Soon-Wook Kim ${ }^{\text {bo }}$, Sug-Whan Kim $^{\text {bp }}$, M. Kleifges ${ }^{\text {ad }}$, P.A. Klimov' ${ }^{\text {, D. Kolev }}{ }^{\text {ai }}$, I. Kreykenbohm ${ }^{\text {bq }}$, K. Kudela ${ }^{\text {ah }}$, Y. Kurihara ${ }^{\mathrm{n}}$, A. Kusenko ${ }^{\text {br, }}$, E. Kuznetsov ${ }^{\mathrm{d}}$, M. Lacombe ${ }^{\mathrm{as}}$, C. Lachaud ${ }^{\mathrm{h}}$, H. Lahmar ${ }^{\mathrm{c}}$, F. Lakhdari ${ }^{\text {aa }}$, O. Larsson ${ }^{\text {ao,am }}$, J. Lee ${ }^{\text {bs }}$, J. Licandro ${ }^{\text {bk }}$, H. Lim ${ }^{\text {bs }}$, L. López Campano ${ }^{\text {az, }}$, M.C. Maccarone ${ }^{1, \mathrm{~m}}$, S. Mackovjak ${ }^{\mathrm{aj}}$, M. Mahdi ${ }^{\mathrm{c}}$, D. Maravilla ${ }^{\mathrm{av}}$, L. Marcelli $^{\mathrm{bt}}$, J.L. Marcos ${ }^{\mathrm{az}}$, A. Marini ${ }^{\mathrm{bb}}$, K. Martens ${ }^{\mathrm{br}}$, Y. Martín ${ }^{\mathrm{bk}}$, O. Martinez ${ }^{\mathrm{bu}}$, G. Masciantonio $^{\mathrm{ap}}$, K. Mase $^{\mathrm{bw}}$, R. Matev ${ }^{\mathrm{ai}}$, J.N. Matthews ${ }^{\mathrm{bx}}$, N. Mebarki ${ }^{\text {bv }}$, G. Medina-Tanco ${ }^{\mathrm{av}}$, L. Mehrad ${ }^{\mathrm{a}}$, M.A. Mendoza ${ }^{\text {by }}$, A. Merino ${ }^{\mathrm{az}}$, T. Mernik ${ }^{\mathrm{u}}$, J. Meseguer, S. Messaoud ${ }^{\mathrm{c}}$, O. Micu ${ }^{\mathrm{o}}$, J. Mimouni $^{\text {bv }}$, H. Miyamoto $^{\mathrm{ac}, \mathrm{v}}$, Y. Miyazaki $^{\mathrm{bz}}$, Y. Mizumoto ${ }^{\mathrm{bm}}$, G. Modestino ${ }^{\mathrm{bb}}$, A. Monaco ${ }^{\mathrm{x}, \mathrm{y}}$, D. Monnier-Ragaigne ${ }^{\mathrm{g}}$, J.A. Morales de los Ríos ${ }^{\mathrm{r}}$, C. Moretto ${ }^{g}$, V.S. Morozenko ${ }^{z}$, B. Mot $^{\text {as }}$, T. Murakami ${ }^{\text {ca }}$, B. Nadji ${ }^{\mathrm{c}}$, M. Nagano ${ }^{\text {bz }}$, M. Nagata ${ }^{\text {cb }}$, S. Nagataki ${ }^{\mathrm{ao}}$, T. Nakamura ${ }^{\mathrm{cc}}$, T. Napolitano ${ }^{\mathrm{bb}}$, A. Nardelli ${ }^{\mathrm{s}}, \mathrm{D}$. Naumov ${ }^{\mathrm{ae}}$, R. Nava ${ }^{\mathrm{av}}$, A. Neronov ${ }^{\text {aj, }}$, K. Nomoto ${ }^{\text {br }}$, T. Nonaka ${ }^{\mathrm{p}}$, T. Ogawa ${ }^{\mathrm{ao}}$, S. Ogio ${ }^{\mathrm{cd}}, \mathrm{H}$. Ohmori ${ }^{\mathrm{ao}}$, A.V. Olinto ${ }^{\text {ay }}$, P. Orleańskiag $^{\text {ag }}$, G. Osteria ${ }^{\text {al }}$, W. Painter ${ }^{\text {ad }}$, M.I. Panasyuk ${ }^{\mathrm{z}}$, B. Panico ${ }^{\text {al }}$, E. Parizot ${ }^{\mathrm{h}}$, I.H. Park ${ }^{\text {bs }}$, H.W. Park ${ }^{\text {bs }}$, B. Pastircak ${ }^{\text {ah }}$, T. Patzak ${ }^{\text {h }}$, T. Paul ${ }^{\mathrm{j}}$, C. Pennypacker ${ }^{\text {aw }}$, M. Perdichizzi ${ }^{\mathrm{s}}$, I. Pérez-

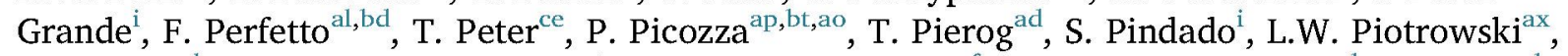
S. Piraino ${ }^{\mathrm{u}, \mathrm{l}}$, L. Placidi ${ }^{\mathrm{s}}$, Z. Plebaniak ${ }^{\mathrm{t}}$, S. Pliego ${ }^{\mathrm{av}}$, A. Pollini ${ }^{\mathrm{af}}$, E.M. Popescu ${ }^{\mathrm{o}}$, P. Prat ${ }^{\mathrm{h}}$, G. Prévôt ${ }^{\mathrm{h}}$, H. Prieto ${ }^{\text {r }}$, M. Putis ${ }^{\text {ah }}$, J. Rabanal ${ }^{g}$, A.A. Radu ${ }^{\circ}$, M. Rahmani ${ }^{\text {aa }}$, P. Reardon ${ }^{\mathrm{d}}$, M. Reyes ${ }^{\mathrm{bk}}$, M. Rezazadeh ${ }^{\mathrm{ay}}$, M. Ricci ${ }^{\mathrm{bb}}$, M.D. Rodríguez Frías ${ }^{\mathrm{r}}$, F. Ronga ${ }^{\mathrm{bb}}$, M. Roth ${ }^{\mathrm{ad}}$, H. Rothkaehl $^{\mathrm{ag}}$, 
G. Roudil $^{\text {as }}$, I. Rusinov ${ }^{\text {ai }}$, M. Rybczyński ${ }^{\text {cf }}$, M.D. Sabau ${ }^{\mathrm{w}}$, G. Sáez Cano ${ }^{\mathrm{r}}$, H. Sagawa ${ }^{\mathrm{P}}$, Z. Sahnoune ${ }^{\text {ba }}$, A. Saito ${ }^{\text {cc }}$, N. Sakaki ${ }^{\text {cd }}$, M. Sakata ${ }^{\text {bl }}$, H. Salazar ${ }^{\text {bu }}$, J.C. Sanchez ${ }^{\text {av }}$, J.L. Sánchez ${ }^{\text {az }}$, A. Santangelo ${ }^{\text {u }}$, L. Santiago Crúz ${ }^{\text {av }}$, A. Sanz-Andrés ${ }^{i}$, M. Sanz Palominow ${ }^{w}$ O. Saprykin ${ }^{\text {be }}$, F. Sarazin ${ }^{\text {ax }}$, H. Sato ${ }^{\text {bl }}$, M. Sato ${ }^{\text {cg }}$, T. Schanz ${ }^{\mathrm{u}}$, H. Schieler ${ }^{\text {ad }}$, V. Scotti ${ }^{\text {al,bd }}$, A. Segreto ${ }^{\text {l,m }}$, S. Selmane ${ }^{\mathrm{h}}$, D. Semikoz ${ }^{\mathrm{h}}$, M. Serra ${ }^{\mathrm{bk}}$, S. Sharakin ${ }^{\mathrm{z}}$, T. Shibata ${ }^{\text {ch }}$, H.M. Shimizu ${ }^{\mathrm{ci}}$, K. Shinozaki ${ }^{\mathrm{u}}$, T. Shirahama ${ }^{\text {bf }}$, G. Siemieniec-Oziẹbło ${ }^{\text {cj }}$, J. Sledd ${ }^{\text {au }}$, K. Słomińska ${ }^{\text {ag }}$, A. Sobey $^{\text {au }}$, I. Stan ${ }^{\circ}$,

T. Sugiyama $^{\text {ci }}$, D. Supanitsky ${ }^{\text {av }}$, M. Suzuki ${ }^{\text {bg }}$, B. Szabelska ${ }^{\mathrm{t}}$, J. Szabelski ${ }^{\mathrm{t}}$, H. Tahi $^{\mathrm{c}}$, F. Tajima ${ }^{\text {ck }}$

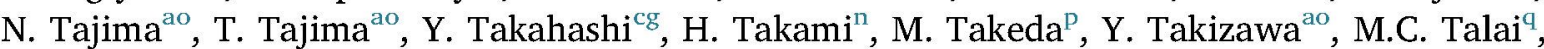

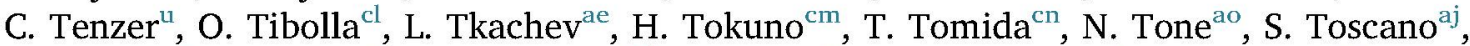

M. Traïche ${ }^{c}$, R. Tsenov ${ }^{\text {ai }}$, Y. Tsunesada ${ }^{\text {cd }}, K$. Tsuno ${ }^{\text {ao }}$, T. Tymieniecka $^{\mathrm{t}}$, T. Uchihori $^{\mathrm{co}}$, M. Unger $^{\text {ad }}$, O. Vaduvescu ${ }^{\text {bk }}$, J.F. Valdés-Galicia ${ }^{\text {av }}$, P. Vallania ${ }^{\text {ac,aq }}$, G. Vankova ${ }^{\text {ai }}$, C. Vigorito ${ }^{\text {ac,v }}$,

L. Villaseñor ${ }^{\mathrm{cP}}$, B. Vlcek ${ }^{\mathrm{r}}$, P. von Ballmoos ${ }^{\mathrm{as}}$, M. Vrabel ${ }^{\mathrm{bc}}, \mathrm{S}$. Wada ${ }^{\mathrm{ao}}, \mathrm{J}$. Watanabe ${ }^{\mathrm{bm}}$,

S. Watanabe ${ }^{\text {cg }}$, J. Watts Jr. ${ }^{\text {d }}$, M. Weber ${ }^{\text {ad }}$, R. Weigand Muñoz ${ }^{\text {az }}$, A. Weindl ${ }^{\text {ad }}$, T.J. Weiler ${ }^{\text {ab }}$,

T. Wibig ${ }^{\mathrm{t}}$, L. Wiencke ${ }^{\mathrm{ax}}$, M. Wille ${ }^{\mathrm{bq}}$, J. Wilms $^{\mathrm{bq}}$, Z. Włodarczyk ${ }^{\mathrm{cf}}$, T. Yamamoto ${ }^{\mathrm{bl}}$, Y. Yamamoto ${ }^{\mathrm{bl}}$, J. Yang ${ }^{\mathrm{cq}}$, H. Yano ${ }^{\mathrm{bg}}$, I.V. Yashin ${ }^{\mathrm{Z}}$, D. Yonetoku ${ }^{\mathrm{ca}}$, S. Yoshida ${ }^{\mathrm{bw}}$, R. Young ${ }^{\mathrm{au}}$, I.S Zgura ${ }^{ }$, M.Yu. Zotov ${ }^{\mathrm{z}}$, A. Zuccaro Marchi ${ }^{\text {ao }}$

a Telecom Lab., Faculty of Technology, University Abou Bekr Belkaid, Tlemcen, Algeria

${ }^{\mathrm{b}}$ Nihon University Chiyoda, Tokyo, Jopan

${ }^{c}$ Centre for Development of Advanced Technologies (CDTA), Algiers, Algeria

d University of Alabama in Huntsville, Huntsville, USA

e Omega, Ecole Polytechnique, CNRS/IN2P3, Palaiseau, France

${ }^{f}$ Laboratory of Theoretical Physics LPT, University of Jijel, Jijel, Algeria

${ }^{g}$ LAL, Univ Paris-SudParis-Sud, CNRS/IN2P3, Orsoy, France

${ }^{h}$ APC, Univ Paris Diderot, CNRS/IN2P3, CEA/Irfu,CEA/Irfu, Obs de Paris, Sorbonne Paris Cité, France

${ }^{i}$ Universidad Politécnia de madrid (UPM), Madrid, Spain

${ }^{\mathrm{j}}$ Lehman College, City University of New York (CUNY), USA

k University of California (UCLA), Los Angeles, USA

${ }^{1}$ INAF - Istituto di Astrofisica Spaziale e Fisica Cosmica di Palermo, Italy

${ }^{\mathrm{m}}$ Istituto Nazionale di Fisica Nucleare - Sesione di Catania, Italy

${ }^{\mathrm{n}}$ High Energy Accelerator Research Organization (KEK), Tsukuba, Japan

Institute of Space Science ISS, Magurele, Romania

P Institute for Cosmic Ray Research, University of Tokyo, Kashiwa, Japan

${ }^{9}$ LPR at Dept. of Physics, Faculty of Sciences, University Badji Mokhtar, Annaba, Algeria

Universidad de Alcalá (UAH), Madrid, Spain

${ }^{s}$ UTIU, Dipartimento di Ingegneria, Rome, Italy

${ }^{\mathrm{t}}$ National Centre for Nuclear Research, Lodz, Poland

1 Institute for Astronomy and Astrophysics, Kepler Center, University of Tübingen, Germany

$\checkmark$ Dipartimento di Fisica, Università di Torino, Italy

${ }^{w}$ Instituto Nacional de Técnica Aeroespacial (INTA), Madrid, Spain

${ }^{\mathrm{x}}$ Istituto Nazionale di Fisica Nucleare - Sezione di Bari, Italy

Università degli Studi di Bari Aldo Moro and INFN - Sezione di Bari, Italy

${ }^{\mathrm{z}}$ Skobeltsyn Institute of Nuclear Physics, Lomonosov Moscow State University, Russia

${ }^{\text {aa }}$ Research Unit on Optics and Photonics, UROP-CDTA, Sétif, Algeria

ab Vanderbilt University, Nashville, USA

ac Osservatorio Astrofisico di Torino, Istituto Nazionale di Astrofisica, Italy

ad Karlsruhe Institute of Technology (KIT), Germany

ae Joint Institute for Nuclear Research, Dubna, Russia

${ }^{\text {af }}$ Swiss Center for Electronics and Microtechnology (CSEM), Neuchâtel, Switzerland

ag Space Research Centre of the Polish Academy of Sciences (CBK), Warsaw, Poland

ah Institute of Experimental Physics, Kosice, Slovakia

ai St. Kliment Ohridski University of Sofia, Bulgaria

af ISDC Data Centre for Astrophysics, Versoix, Switzerland

${ }^{\text {ak }}$ Universidad Autónoma de Chicpas (UNACH), Chiapas, Mexico

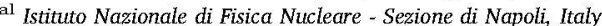

${ }^{\text {am }}$ KTH Royal Institute of Technology, Stockholm, Sweden

an Dipartimento di Fisica e Astronomia - Università di Catania, Italy

ao RIKEN, Wako, Japan

ap Istituto Nazionale di Fisica Nucleare - Sezione di Roma Tor Vergata, Italy

aq Istituto Nazionale di Fisica Nucleare - Sezione di Torino, Italy

ar Consiglio Nazionale delle Ricerche (CNR) - Istituto di Fisica Applicata Nello Carrara, Firenze, Italy

as IRAP, Université de Toulouse, CNRS, Toulouse, France

at Kinki University, Higashi-Osaka, Japan

au NASA - Marshall Space Flight Center, USA

av Universidad Nacional Autónoma de México (UNAM), Mexico

aw Space Science Laboratory, University of Califormia, Berkeley, USA

ax Colorado School of Mines, Golden, USA

ay University of Chicago, USA

${ }^{a z}$ Universidad de León (ULE), León, Spain

ba Dep. Astronomy, Centre Res. Astronomy, Astrophysics and Geophysics (CRAAG), Algiers, Algeria

bb Istituto Nazionale di Fisica Nucleare - Laboratori Nazionali di Frascati, Italy

${ }^{\text {bc }}$ Technical University Kosice (TUKE), Kosice, Slovakia 
bd Università di Napoli Federico II - Dipartimento di Scienze Fisiche, Italy

be Central Research Institute of Machine Building, TsNIIMash, Korolev, Russia

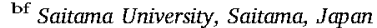

${ }^{b g}$ Institute of Space and Astronautical Science/JAXA, Sagamihara, Japan

bh DIETI, Università degli Studi di Napoli Federico II, Napoli, Italy

bi Institute for Space-Earth Environmental Research, Nagoya University, Nagoya, Japan

${ }^{b j}$ Experimental Physics Institute, Kepler Center, University of Tübingen, Germany

${ }^{\mathrm{bk}}$ Instituto de Astrofisica de Canarias (IAC), Tenerife, Spain

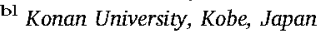

bm National Astronomical Observatory, Mitaka, Jopan

bn Department of Physics, Faculty of Sciences, University of M'sila, Algeria

bo Korea Astronomy and Space Science Institute (KASI), Daejeon, Republic of Korea

bP Center for Galcxy Evolution Research, Yonsei University, Seoul, Republic of Korea

bq ECAP, University of Erlangen-Nuremberg, Germany

br University of Tokyo, Tokyo, Japan

bs Sungkyunkwan University, Seoul, Republic of Korea

bt Università di Roma Tor Vergata - Dipartimento di Fisica, Roma, Italy

bu Benemérita Universidad Autónoma de Puebla (BUAP), Mexico

${ }^{b v}$ Lab. of Math and Sub-Atomic Phys. (LPMPS), Univ. Constantine I, Constantine, Algeria

bw Chiba University, Chiba, Japan

bx University of Utah, Salt Lake City, USA

by Centro de Desarrollo Aeroespacial - Instituto Politécnico National (CDA-IPN), Mexico

bz Fukui University of Technology, Fukui, Japan

ca Kanazawa University, Kanazawa, Japan

${ }^{\mathrm{cb}}$ Kobe University, Kobe, Japan

cc Kyoto University, Kyoto, Japan

cd Graduate School of Science, Osaka City University, Jcpan

ce Institute for Atmospheric and Climate Science, ETH Zürich, Switzerland

ef Jan Kochanowski University, Institute of Physics, Kielce, Poland

${ }^{c g}$ Hokkaido University, Sapporo, Japan

ch Aoyama Gakuin University, Sagamihara, Japan

${ }^{c i}$ Nagoya University, Nagoya, Japan

cj Jagiellonian University, Astronomical Observatory, Krakow, Poland

ck Hiroshima University, Hiroshima, Japan

${ }^{\mathrm{cl}}$ Centro Mesoamericano de Física Teórica (MCTP), Mexico

cm Interactive Research Center of Science, Tokyo Institute of Technology, Tokyo, Japan

cn Shinshu University, Nagano, Japan

${ }^{c o}$ National Institute of Radiological Sciences, Chiba, Japan

cp Universidad Michoacana de San Nicolas de Hidalgo (UMSNH), Morelia, Mexico

${ }^{c q}$ Ewha Womans University, Seoul, Republic of Korea

\section{A R T I C L E I N F O}

\section{Keywords}

Meteors

Nuclearites

JEM-EUSO

Mini-EUSO

EUSO-TA

\begin{abstract}
A B S T R A C T
We summarize the state of the art of a program of UV observations from space of meteor phenomena, a secondary objective of the JEM-EUSO international collaboration. Our preliminary analysis indicates that JEMEUSO, taking advantage of its large FOV and good sensitivity, should be able to detect meteors down to absolute magnitude close to 7 . This means that JEM-EUSO should be able to record a statistically significant flux of meteors, including both sporadic ones, and events produced by different meteor streams. Being unaffected by adverse weather conditions, JEM-EUSO can also be a very important facility for the detection of bright meteors and fireballs, as these events can be detected even in conditions of very high sky background. In the case of bright events, moreover, exhibiting some persistence of the meteor train, preliminary simulations show that it should be possible to exploit the motion of the ISS itself and derive at least a rough 3D reconstruction of the meteor trajectory. Moreover, the observing strategy developed to detect meteors may also be applied to the detection of nuclearites, exotic particles whose existence has been suggested by some theoretical investigations. Nuclearites are expected to move at higher velocities than meteoroids, and to exhibit a wider range of possible trajectories, including particles moving upward after crossing the Earth. Some pilot studies, including the approved Mini-EUSO mission, a precursor of JEM-EUSO, are currently operational or in preparation. We are doing simulations to assess the performance of Mini-EUSO for meteor studies, while a few meteor events have been already detected using the ground-based facility EUSO-TA.
\end{abstract}

\section{Introduction}

For the past several years an International Collaboration involving several research institutes located in 16 countries of 4 different continents (Europe, Asia, America and Africa) has been working on the development of the JEM-EUSO (Extreme Universe Space Observatory on board the Japanese Experiment Module) mission
(Adams et al., 2015a), whose main idea is to put a 2-m class telescope aboard the International Space Station (ISS), to carry out nadir-oriented observations of a variety of very rapid transient physical phenomena.

The project has evolved with time, and it now also includes smaller, pathfinder missions, namely experiments designed to test the observational strategy, and validate the existing technology to be used for the main mission. Among these JEM-EUSO precursors, there are experi- 
ments carried out at the EUSO-TA facility (Adams et al., 2015c) and a recently approved ISS-based mission, named Mini-EUSO (Ricci et al., 2015).

The main goal of JEM-EUSO is the detection of Extreme Energy Cosmic Rays (EECR, characterized by energy $E$ above $5 \times 10^{19} \mathrm{eV}$ ), making use of a dedicated refractive telescope having a $2.5 \mathrm{~m}$ diameter aperture equipped with an UV detector covering a wavelength interval between 290 and $430 \mathrm{~nm}$. The telescope will be positioned in one of the modules of the ISS, and will carry out nadir observations from a height of about $400 \mathrm{~km}$ above sea level with a full Field of View (FOV) of $\sim 60^{\circ}$. In this configuration, the instrument will detect the secondary light emissions induced by cosmic rays in the atmosphere (fluorescence and Cherenkov light). This mission design also makes possible the detection of a variety of transient luminous events in the atmosphere (Adams et al., 2015d), including meteor phenomena (Adams et al., 2015e).

The pathfinder mission Mini-EUSO will use the same technologies of JEM-EUSO, but with a smaller aperture ( $25 \mathrm{~cm}$ diameter), resulting in a much coarser spatial resolution and a smaller FOV $\sim 40^{\circ}$. Mini-EUSO is currently an approved project of ASI and ROSCOSMOS, and will be put aboard in the Russian Service Module of the ISS, pointing in the nadir direction through an UV transparent window. The scientific objectives of Mini-EUSO range from cosmic rays to planetary and atmospheric science, including tests of space observation of meteors and fireballs at UV wavelengths.

In parallel to this space program, a ground-based detector, EUSOTA, has been operating since 2013 at the Telescope Array facility in Utah. Its main purpose is to perform ground-based tests of the planned observational technique and instrumentation of JEM-EUSO. During preliminary campaigns of cosmic ray observation, a few meteor events have been already detected, confirming the expectations.

The paper is organized as follows. Section 2 and 3 will briefly report on the concept of the JEM-EUSO and Mini-EUSO detectors and their expected performance for meteor and nuclearite detection, the latter being exotic particles which have been suggested by some theoretical investigations to address the dark matter issue (De Rujula and Glashow, 1984). Section 4 will summarize the ongoing efforts to implement into ESAF (Berat et al., 2010), the software package employed by the JEMEUSO collaboration, the basic properties of meteor phenomena and the necessary procedures for their detection with the JEM-EUSO and MiniEUSO instruments. Section 5 shows the first examples of meteor tracks detected by means of the JEM-EUSO observational approach, using the EUSO-TA facility in Utah.

\section{JEM-EUSO}

An extensive review of the JEM-EUSO concept, including detailed descriptions of the instrument hardware, has been published in a special issue (Volume 40, November 2015) of the journal Experimental Astronomy. Here, we briefly recall only the essential elements.

The system consists of four major parts: (1) light-collecting optics; (2) focal surface detector; (3) on-board electronics; (4) lodgement structure. In practical terms, JEM-EUSO can be thought of as a large aperture and extremely-fast digital camera with a large field of view, specifically designed to detect extremely rapid events of light emission at UV wavelengths. As mentioned above, the optics consists of a telescope having an aperture of about $2.5 \mathrm{~m}$ and a field of view of $60^{\circ}$. The detector is a $\sim 3 \times 10^{5}$ pixels camera which is able to record incoming radiation at a rate of $400 \mathrm{kHz}$. The whole system operates at wavelengths between 290 and $430 \mathrm{~nm}$, in both single-photon-counting and charge integration modes.

Two curved double-sided Fresnel lenses having an external diameter around $2.5 \mathrm{~m}$, a precision middle Fresnel lens and a pupil, constitute the optics of the telescope. The Fresnel lenses provide large-aperture, wide FOV optics with low mass and high UV light transmittance. The resulting angular resolution is $0.075^{\circ}$. This corresponds to a linear size of about $550 \mathrm{~m}$ on the ground (at nadir) We remind that the ISS orbits at a height of about $400 \mathrm{~km}$. The corresponding resolution for observation of sources located at a height of $100 \mathrm{~km}$ above the ground, typical of meteor events, is about $400 \mathrm{~m}$.

The incident UV photons are focused by the optics into the focal surface, where the detector converts them into electric pulses. The focal surface has a spherical shape, about $2.3 \mathrm{~m}$ in diameter with a curvature radius of about $2.5 \mathrm{~m}$. On this surface, about 5000 multi-anode photomultiplier tubes (MAPMT) Hamamatsu R11265-03-M64 (1 in. in size, with $8 \times 8$ pixels) are located. The detector consists of PhotoDetector Modules (PDMs), each one including Elementary Cells (ECs) consisting of 4 units of MAPMT. A total of 137 PDMs are arranged on the focal surface. A Cockcroft-Walton-type high-voltage supply is used to reduce power consumption. It includes a circuit to protect the photomultipliers from sudden and intense light bursts, such as lightning or bright fireball phenomena (Adams et al., 2015b).

The on-board electronics records the signals generated by UV photons. A front-end Application-Specific Integrated Circuit (ASIC) fulfills both functions of single photon counting and charge integration in a 64-channels chip. The electronics are configured in three levels corresponding to the structure of the focal surface detector: front-end electronics at EC level, PDM electronics common to 9 EC units, and focal surface electronics to control 137 units of PDMs. The anode signals from the MAPMT are digitized at time intervals (named Gate Time Units, GTU) of $2.5 \mu \mathrm{s}$ and held in ring memory for an event to trigger the onboard software. After triggering, the data are sent to control boards. JEM-EUSO uses a hierarchical trigger method to reduce the data rate of $\sim 10 \mathrm{~GB} / \mathrm{s}$ down to $297 \mathrm{kbps}$, required for transmission from the ISS to the ground operation center.

In this way, the pulses produced by incident UV photons are read and recorded by the on-board electronics. Whenever a signal pattern is detected, it triggers the start of a sequence of recording operations which involve a selected pixel region on the detector. The recorded signal data are eventually transmitted to the ground operation center.

\subsection{Meteors}

As mentioned above, JEM-EUSO is designed to observe at UV wavelengths. A few preliminary comments seem useful. There are not so many studies of meteors observed in the UV, because observations are usually done at visible wavelengths. An interesting, but unfortunately still rare exception is represented by the detection of a meteor of absolute magnitude around -2 observed during the 1997 Leonid shower by the MSX satellite (Jenniskens et al., 2002). In that case, the meteor could be observed by detectors covering an interval of wavelengths from UV to near-IR. Another example of Leonid meteor spectra in the UV-visible region, using HDTV spectroscopy from an airplane, has been published by Kasuga et al. (2005).

According to available evidence, a meteor spectrum is mostly an emission line spectrum consisting of different components, including: (1) a warm $\approx 4500 \mathrm{~K}$ component of ablation products in the meteor wake, rich in low excitation lines by metal atoms, mainly including $\mathrm{Fe}$, $\mathrm{Mg}, \mathrm{Na}, \mathrm{Ca}$. (2) a hotter $(\approx 10,000) \mathrm{K}$ component from the front of the shock wave. This is rich in lines produced by ions of $\mathrm{Ca}, \mathrm{Mg}$ and other elements. (3) an air plasma component rich in first positive bands of $N_{2}$, $\mathrm{O}, N$. The relative ratio of the above components is variable, depending on the particular ablation conditions experienced by any given meteoroid, including speed, ablation rate, presence of flares.

By considering an UV detector covering the interval of wavelengths of JEM-EUSO, and assuming as usual a V band centered at $550 \mathrm{~nm}$, we can expect, as a first approximation, that the flux in the two bands should be comparable, as both UV and V are dominated by $\mathrm{Mg}$, Fe, and $\mathrm{Na}$ emission from the warm component. This prediction looks reasonable even taking into account that Na sometimes shows differential ablation and can vary among different meteors. Moreover, the V-band can also exhibit some air plasma emission from the first positive band of 
$N_{2}$, which can cause some variations. Based also on the analysis of a few published spectra, we believe that we should not expect the flux from meteors observed in the range of wavelengths covered by JEM-EUSO to be fainter than the flux at visible wavelengths. By taking into account the emission of some Fe and $\mathrm{Mg}$ lines at wavelengths between 350 and $400 \mathrm{~nm}$, we could even expect the UV flux to be brighter than in the V, perhaps by more than one magnitude. This is a subject certainly requiring further investigations. Another interesting field of investigation needing further efforts is a study of the possible UV emission of elements including $C$, which is thought to be abundant in comets, whose emissions in the visible are negligible. In the following, we assume that the meteor fluxes in the wavelength interval covered by JEM-EUSO are comparable to the usual fluxes recorded in the $\mathrm{V}$.

The main results of a preliminary investigation of the expected performance of JEM-EUSO where meteor detection is concerned, including also a study of the best possible observation strategy to optimize the results, have been published by Adams et al. (2015e). Here, we will briefly summarize the current situation, while interested readers will find more details in the above-mentioned paper. As for our reference source for meteor photometric properties, see Pecina and Koten (2009).

The analyses have been based on a simulation software developed to mimic the expected signals produced by meteors on the focal plane of JEM-EUSO in a wide range of possible situations. In particular, any given phenomenon is described by a number of free parameters, including the beginning height of the meteor, the modulus and direction of its velocity vector, the duration of the event, and the morphology of the light-curve produced by the ablation of the meteoroid during its passage through the atmosphere. The simulator is very simple, and no deceleration of the meteoroid has been taken into account so far. Since the photometric behaviour of meteors is very variable and essentially unpredictable a priori, the software has been written in such a way as being able to produce a large variety of possible light-curves, the signal being approximated by a polynomial of 9th degree. Real meteors, especially the brightest ones, often exhibit the presence of one or more flares along the trajectory. Unfortunately, it is not possible to include in a simple simulator all the huge variety of possible situations. In its current version, the meteor simulator allows for the presence of only one possible light flare, and takes as other free parameters the time of occurrence of this flare, its light-curve morphology and duration. In practical terms, the simulations performed so far have assumed some simple light-curve morphology for both the main event and the flare, both being approximated by a polynomial characterized by a raise to a maximum, and subsequent decay. The maximum of the light-curve is another free parameter of the numerical simulator. The computation of the signal is performed by considering two possible observers: one, located at a fixed site on the ground, and another aboard the moving ISS. The varying distances between the meteor and each observer are taken into account in computing the number of photons produced on the focal plane at each instant. In particular, the simulator computes the received signal at time steps of
$1 \mathrm{~ms}$. The number of UV photons, computed by taking into account a fixed conversion from apparent UV magnitudes to photon flux, as well as the location of the received signal on the focal plane, is also computed at each time step.

The simulator described above is very simple, yet it allows us to make several useful computations. For example, it makes it possible to simulate the behaviour of light sources on the terrestrial surface (simulated as meteors at an height of zero $\mathrm{km}$ and with zero velocity).

In terms of meteor measurements, the results of the simulated signals have suggested a general strategy, based on the idea of using different data processing modes, depending on the apparent luminosity of the event, which can vary over a very large interval. Two main techniques for signal measurement are possible, namely photon counting and charge-integration. Photon counting is the standard procedure for the measurement of cosmic ray events, but it is not suitable to record events producing more than a few tens of photo-electrons per pixel per gate time unit (GTU). At higher rates, charge integration becomes the only possible option (Adams et al., 2015e). Note that this is a choice to be made during the phase of data reduction. The on-board processing, once it is automatically triggered, either by photon-counting or by charge-integration, will in any case acquire data both in photon counting as well as in charge-integration mode. Simulations performed so far, including also a number of blind tests, in which the reconstruction of the detected signal was performed without any a priori knowledge of the properties of the simulated event, have shown that a satisfactory reconstruction of the true signals, starting from the recorded photo-electron counts obtained in a large variety of situations, can be achieved.

According to the results obtained so far, and summarized in Table 1 , (where we also include some results corresponding to the Mini-EUSO case, see Section 3) we can conclude that JEM-EUSO should be able to detect meteors as faint of absolute magnitude around +7 , against a dark background. Note that the typical UV background (without Moon) is about 500 photons $/ \mathrm{m}^{2} / \mathrm{ns} / \mathrm{sr}$, which corresponds to 1 count/pixel/ GTU (Adams et al., 2013). According to Adams et al. (2015e), by taking into account the ISS motion and the correspondingly variable role played by anthropogenic light sources (like cities), and natural phenomena such as lightnings and Moon phase, the above conditions are met on the average of $20 \%$ of the time. The results shown in Table 1 include the presence of UV background and a simulation of the trigger logic. In conditions of full Moon, the UV background increases up to 30 count/pixel/GTU. These conditions are met on the average in 35\% of the time and are still acceptable for brighter meteors. A very interesting result was obtained for the case of very bright meteor events, or fireballs. In particular, fireballs can be so bright to be observable in conditions of very bright background, with the brightest events being visible on the dayside. These events are particularly interesting because they involve meteoroids having masses larger than tens of grams. They are so bright that for them a persistent meteor train can be produced, unlike the case of fainter meteors. As a consequence, many points of the trajectory can be observed for consecutive time steps on the focal plane

Table 1

For different values of absolute magnitude in visible light, the Table lists the corresponding flux in the $U$-band (according to the Flux Density Converter of the Spitzer Science Center available at web site http//ssc.spitzer.caltech.edu/warmmission/propkit/pet/magtojy/index.html), the corresponding numbers of photons per second (assuming that the meteor is located at a height of $100 \mathrm{~km}$ and is observed by the ISS in the nadir direction), and the corresponding number of photo-electrons per GTU, for the cases of JEM-EUSO and Mini-Euso, respectively. The corresponding typical mass of the meteor, and the number of events expected to be observed by JEM-EUSO (by assuming a duty cycle of 0.2 ) and by Mini-EUSO are also shown. The relationship between mass and magnitude has been obtained following Robertson and Ayers (1968).

\begin{tabular}{|c|c|c|c|c|c|c|c|}
\hline $\begin{array}{l}\text { Abs. } \\
\text { mag }\end{array}$ & $\begin{array}{l}\text { U-band flux } \\
\left(\mathrm{erg} / \mathrm{s} / \mathrm{cm}^{2} / \mathrm{A}\right)\end{array}$ & $\begin{array}{l}\text { photons } \\
\left(\mathrm{s}^{-1}\right)\end{array}$ & $\begin{array}{l}\text { photo-e } \\
\text { GTU }^{-1} \\
(\mathrm{JEM})\end{array}$ & $\begin{array}{l}\text { photo-e } \\
\text { GTU }^{-1} \\
\text { (Mini) }\end{array}$ & $\begin{array}{l}\text { mass } \\
(\mathrm{g})\end{array}$ & $\begin{array}{l}\text { event } \\
\text { rate } \\
\text { (JEM) }\end{array}$ & $\begin{array}{l}\text { event } \\
\text { rate } \\
\text { (Mini) }\end{array}$ \\
\hline+7 & $6.7 \cdot 10^{-12}$ & $4.3 \cdot 10^{7}$ & 4 & 0.04 & $2 \cdot 10^{-3}$ & $1 / \mathrm{s}$ & $0.4 / \mathrm{s}$ \\
\hline+5 & $4.2 \cdot 10^{-11}$ & $2.7 \cdot 10^{8}$ & 23 & 0.23 & $10^{-2}$ & $6 / \min$ & $2.4 / \mathrm{min}$ \\
\hline 0 & $4.2 \cdot 10^{-9}$ & $2.7 \cdot 10^{10}$ & 2300 & 23 & 1 & 0.27 /orbit & 0.11 /orbit \\
\hline-5 & $4.2 \cdot 10^{-7}$ & $2.7 \cdot 10^{12}$ & $2.3 \cdot 10^{5}$ & 2300 & 100 & $6.3 /$ year & $2.5 /$ year \\
\hline
\end{tabular}


from the ISS, with a gradual drift across the FOV due to the motion of the ISS. So, for these objects, a 3D reconstruction of the trajectory becomes possible, by means of some simple trigonometric computations. This also means it will be possible to compute the original heliocentric orbit of the meteoroid and, in case of objects sufficiently massive to reach the ground, to constrain the region where the freshly fallen meteorite should be searched for. This is an extremely important result for the study of the origin, composition, and evolution of these celestial bodies.

We did some preliminary computations of the reconstruction of the 3D trajectory of a meteor by measuring the recorded drift of the signal corresponding to three points of the meteor train. The results suggest that, in the case of meteors having an absolute magnitude of the order of +3 and brighter, the modulus of the velocity vector of the meteor can be computed with an accuracy of the order of $15 \%$ or better. The corresponding 3D coordinates of the meteor head can also be estimated with a similar accuracy, the best results depending on the choice of the brightest points of the meteor track, which produce a longer drift on the focal plane. This is shown in Fig. 1, in which four different damping coefficients of the meteor train are considered, taken from the cases of some fireballs published by Shrbený and Spurný (2009). The choice of a meteor having a magnitude of +3 is made just as an example, and it is not critical for our purposes. The possibility to compute the 3D trajectory depends on the capability to detect a persistent meteor train. In principle, the computation would depend on the damping coefficient, only, and not on the magnitude. The reason is that the variable gain of the photomultipliers is designed in such a way that in any condition of
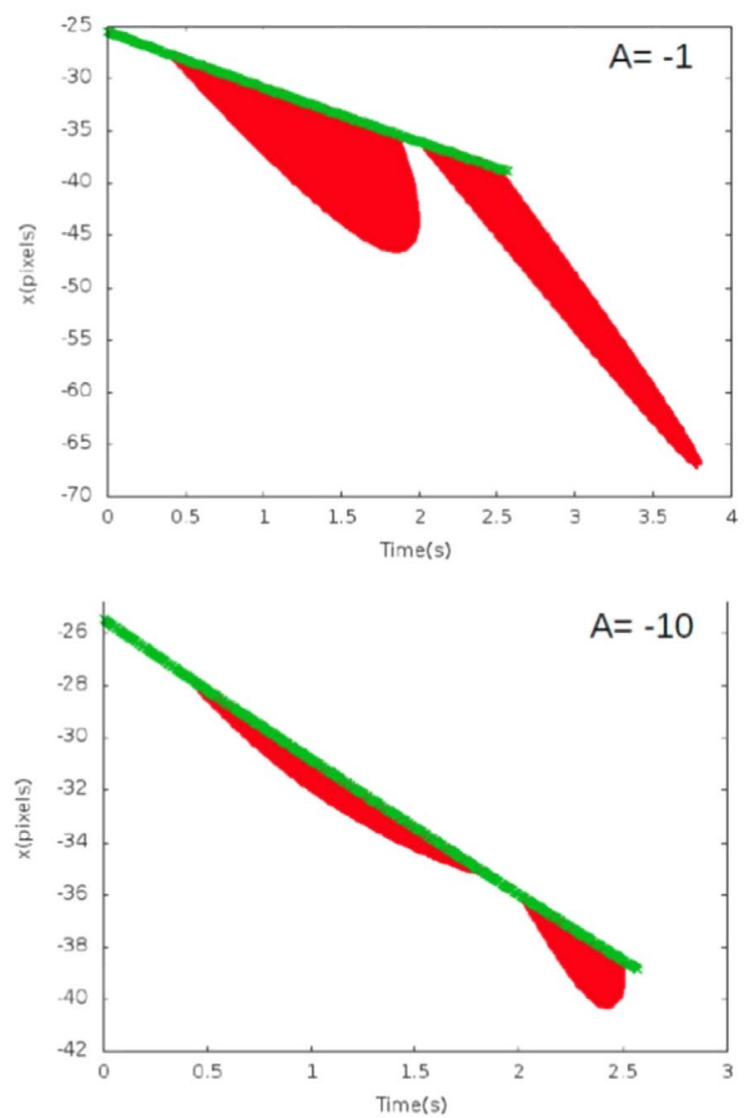

illumination the number of recorded photo-electrons varies in an identical range (between 1 and 100 counts/pixel/GTU). Therefore, our example referring to a $M=+3$ meteor would be equivalent to the case of a meteor of $M=-2$, corresponding to activation of the first protection level. Of course, in the case of such a bright signal the background flux would be close to zero. The ratio between the flux of the meteor head and the persistent meteor train depends at each instant only upon the damping parameter. In the real world, however, the magnitude of the meteor plays also an essential role for two reasons: (1) the meteor flux must be much higher than the background signal. (2) As a matter of fact, we know that faint meteors do not produce observable meteor trains.

\subsection{Nuclearites}

Another possible application of JEM-EUSO is the detection of completely different, but extremely interesting luminous sources, the so-called nuclearites. As explained by Adams et al. (2015e), the possible existence of nuclearites, which could be constituents of dark matter, is predicted by some theoretical studies (De Rujula and Glashow, 1984). Nuclearites are thought to be nuggets made of massive nuclear particles including strange quarks among their constituents. This nuclear matter would be expected to have a small positive electric charge, but neutrality should be assured by the presence of a cloud of surrounding, captured electrons (see, again, De Rujula and Glashow, 1984). According to Adams et al. (2015e), the absolute magnitude $M$ of nuclearites should depend only on their mass and should be constant,
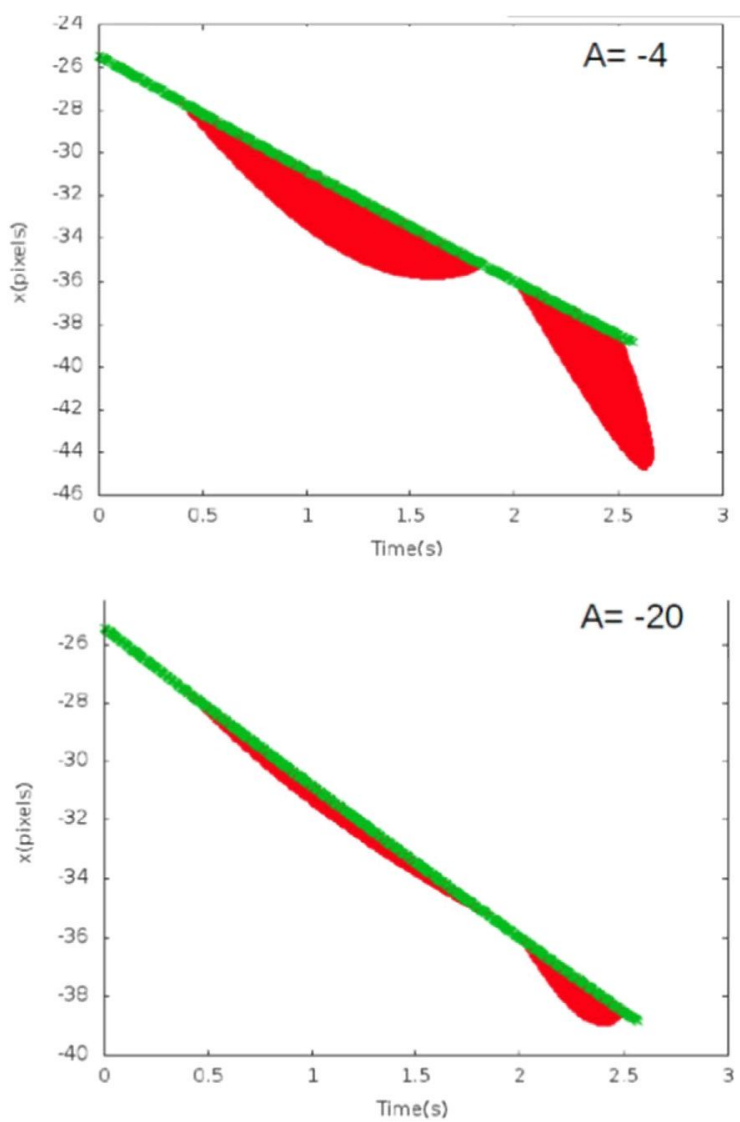

Fig. 1. Example of a bright meteor (absolute magnitude $M=3$ at maximum brightness) producing different signals on the focal plane, depending on the assumed persistence of the meteor train. It is assumed that each point of the meteor train has an intensity decaying according to the relation $I(t)=I_{0} \cdot e^{A t}$, with $A$ being a damping coefficient expressed in units of $s^{-1}$. The Figure shows the expected behaviour for four possible values of $A$, indicated in each panel. The simulated light-curve is assumed to exhibit a strong flare. The green line in the four panels shows the $x$-axis location of the meteor head measured in pixels on the focal plane, as a function of time (the reference frame is centered at the center of the focal plane, the $x$ and $y$ axes being horizontal and mutually perpendicular, while the $z$ axis is oriented toward the nadir direction). The length of the green line is identical in the four cases. The scale of the different plots changes to include the varying domain of the position versus time plane occupied by the drifting signal (in red) produced by the meteor train, depending on the chosen value of the damping coefficient. 
according to the relation:

$M=15.8-1.67 \log (m)$

where $m$ is the mass expressed in $\mu \mathrm{g}$. The emitted energy should follow a black-body relation, ensuring observable fluxes in the interval of wavelengths covered by JEM-EUSO. Also interesting is the prediction that the light flux should be emitted only up to a maximum height $h_{\max }$, which is mass-dependent. According to expectations, most nuclearites should emit only a faint radiation at heights not exceeding a few $\mathrm{km}$, but very massive nuclearites might exist, having masses up to $10^{4} \mathrm{~g}$. Such objects could be visible up to about $60 \mathrm{~km}$ of height, and have absolute magnitudes $M<0$.

Nuclearites are expected to move at galactic speeds, up to $570 \mathrm{~km} / \mathrm{s}$, significantly higher than meteor velocities, but still in the range of slow events for the JEM-EUSO detector. The big difference with respect to meteors is that nuclearites more massive then $0.1 \mathrm{~g}$ should be able to have the possibility to pass freely through the Earth, so some of them, in particular the most massive ones, could be even observed as very fast meteor-like events moving upward. In spite of the absence in the literature of records of meteor events having such anomalous trajectories, and taking also into account the large nuclear masses required to produce detectable events, the possible observation of nuclearites by JEM-EUSO remains a fairly remote, but nevertheless extremely exciting possibility. JEM-EUSO is sensitive to strangelet nuclearites with mass $m>10^{22} \mathrm{GeV}$ (see Fig. 2). A null observation of these events will place orders of magnitude more stringent limits than current flux limits (Adams et al., 2015e).

\section{The Mini-EUSO pilot mission and meteor observation}

As mentioned above, Mini-EUSO is an approved pilot mission aboard the ISS, to test the main mission concepts developed for JEMEUSO. Originally born as a joint project between the national scientific communities of Italy and Russia, Mini-EUSO was selected in Italy by the Italian Space Agency (ASI) and under the name "UV atmosphere", it was approved by the Russian Space Agency (ROSCOSMOS) and included in the long-term program of ISS-based space experiments.

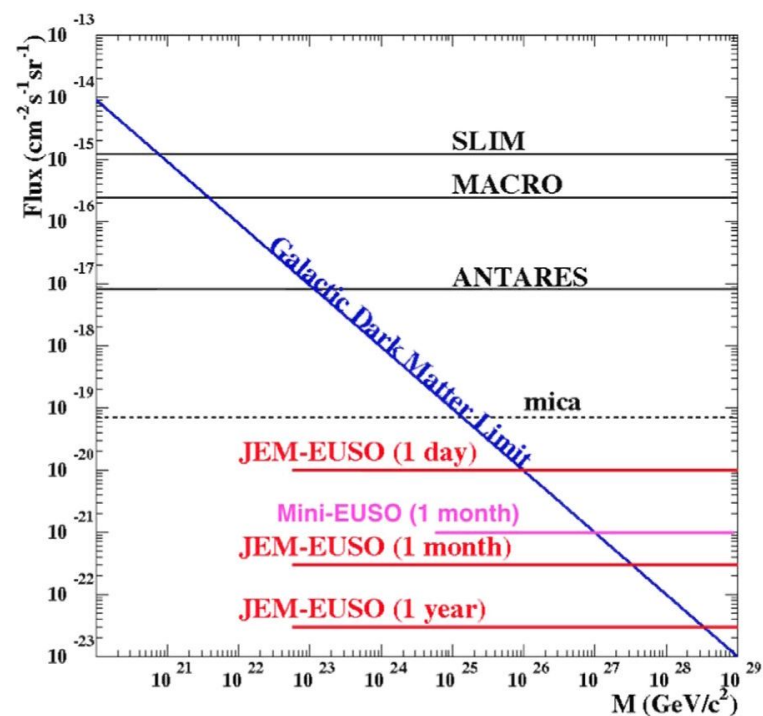

Fig. 2. The JEM-EUSO $90 \%$ confidence level upper limit on the flux of nuclearites resulting from null detection over $24 \mathrm{~h}, 1$ month and 1 year of JEM-EUSO operations. The limit for Mini-EUSO is reported only for $\mathbf{1}$ month operation. For the other durations, they can be rescaled similarly from JEM-EUSO ones. The limits of other experiments MACRO (Ambrosio et al., 2000), SLIM (Cecchini et al., 2008), ANTARES (Pavalas et al., 2012), mica (Price, 1988) (the latter being dependent on several additional assumptions) are also shown for a comparison.
Currently, Mini-EUSO is an established project in the framework of the whole JEM-EUSO Collaboration.

The Mini-EUSO instrument, whose scheme and conceptual design are shown in Fig. 3, is composed of one single element of the basic detection unit conceived in the JEM-EUSO design, namely the Photo Detector Module (PDM). It consists of 36 Hamamatsu Multi Anode Photo Multiplier Tubes (MAPMT M64), 64 pixels each, for a total of 2304 pixels. The optical system consists of two Fresnel lenses, both having a diameter of $25 \mathrm{~cm}$. The front-end electronics are based on the same-photon counting technique developed for JEM-EUSO. According to current plans, two visible cameras should also be installed as ancillary instruments to complement the UV detections performed by the Mini-EUSO focal assembly. The Mini-EUSO instrument will be inserted in a specific container, a mechanical box providing also all the needed interfaces to the transparent, nadir looking UV window of the Zvezda Russian module aboard the ISS.

The FOV of Mini-EUSO will be about $\pm 20^{\circ}$, corresponding to a radius at ground of $\sim 150 \mathrm{~km}$, with $\sim 0.8^{\circ}$ per pixel. The trigger system and data acquisition is organized in different time scales. For meteor observation purposes, Mini-EUSO will continuously record data with $40 \mathrm{~ms}$ time resolution. Data are saved on disks aboard the ISS, and periodically brought to the ground by astronauts for data reduction. Meteor detection and tracking will be performed on ground after data recovery. However, triggers on board will also allow portions of the tracks to be recorded with time resolutions $2.5 \mu \mathrm{s}$ and $320 \mu \mathrm{s}$, respectively. The same simulator used to evaluate JEM-EUSO performance has been adapted to the Mini-EUSO instrument to get a first estimation of its performance. Thanks to the possibility of performing data reduction on ground, Mini-EUSO can deal with meteors having magnitudes around +4 and +5 in optimal dark-background conditions. In such conditions the signal, integrated at steps of $40 \mathrm{~ms}$ will exceed by 3-4o the expected UV-nightglow level. Every year, Mini-EUSO will be able to collect a number of meteor signals that should be three times less than that expected for JEM-EUSO. The quality of the recorded meteor data will be also worse as the FOV per pixel is 10 times coarser compared to JEM-EUSO. However, in spite of these limitations, it will still be possible to demonstrate the observation principles conceived for meteor observations by JEM-EUSO.

Regarding the observation of nuclearites, the limits for null observation at a first order of approximation can be rescaled from JEM-EUSO results by simply taking into account both the ratio of the apertures, which per se shifts the limit on the detectable mass size of the nuclearite by two orders of magnitude, and the ratio of the FOV, which decreases the rate of events by a factor $\sim 2.5$ (see Fig. 2). Also, the quality of the meteor path determination will be worse in Mini-EUSO, but thanks to the higher speed of these objects the total number of

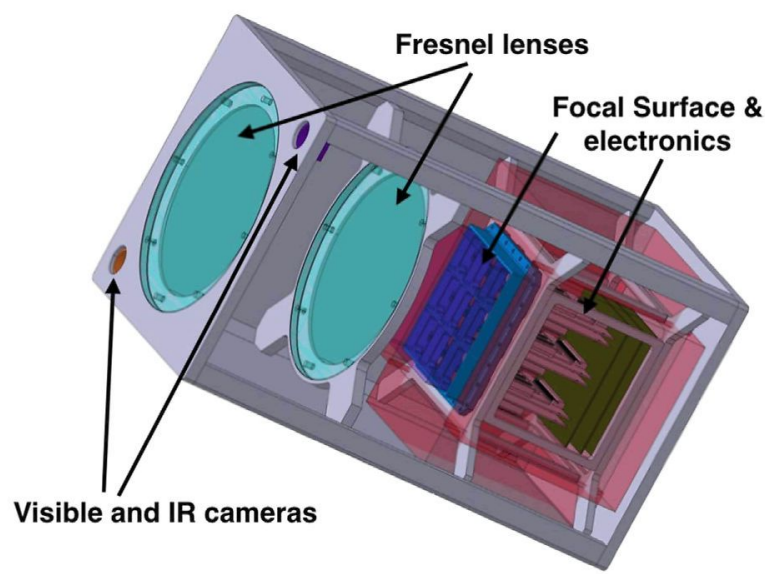

Fig. 3. A three dimensional representation of the Mini-EUSO instrument payload 
pixels crossed by the signal will increase by a factor of a few, so improving considerably the quality of the recorded path compared to the case of a meteor moving along the same direction.

\section{Implementation of meteor simulations in ESAF}

The performance of JEM-EUSO and Mini-EUSO has been so far evaluated using a simple numerical simulator of meteor phenomena, as described in Section 2. Currently, there is an on-going effort to include this already developed software in the ESAF (EUSO Simulation and Analysis Software) package. The reason is that ESAF is the official software tool to perform simulations of extended air shower (EAS) development of cosmic ray, photon production and transport through the atmosphere, and detector response for optics and electronics. Moreover, ESAF includes also algorithms and tools for the reconstruction of the properties of air showers produced by extremely energetic cosmic rays. Originally developed for the EUSO-ESA mission, all the necessary steps have been taken in recent times to implement in ESAF all the planned JEM-EUSO, Mini-EUSO and EUSO-TA instrumental configurations, in order to assess the full range of expected performances for cosmic ray observation (Bertaina et al., 2014). It is therefore important to implement in ESAF also the possibility to carry out detailed simulations of much slower events, taking into account the large variety of possible signals produced by meteors.

In fact, cosmic ray phenomena (typically a hundred $\mu$ s) are much shorter in time than meteor phenomena, lasting up to a few seconds (see Table 2 for a comparison of the main characteristics). The typical signal expected for a $10^{20} \mathrm{eV}$ extensive air shower is of the order of a few thousands of photons in a hundred $\mu$ s. For each photon it is necessary to track the trajectory through the detector with $n s$ time resolution. Such brightness on the same time scale corresponds to a meteor of magnitude $M=+5$. However, in the case of meteors, the signal duration is expected to be around 3 orders of magnitude longer. The requirements in terms of run-time memory and data output size are therefore in principle much more severe. Therefore, it is important to adapt ESAF to the simulation of much longer events, lasting up to a few seconds. So far, the way to overcome the problem has been to simulate the measurement of only a few points of the meteor trajectory to follow its evolution. A detailed explanation of the method is reported in Nardelli (2014). Moreover, ESAF now also includes an implementation of a formula by Jacchia et al. (1967) which links the magnitude, mass and the velocity of the meteoroid. Given the meteor's velocity and magnitude, that are free parameters in the simulations, one can therefore derive the corresponding mass of the meteoroid. By assuming a value of the density $\rho$ (so far a fixed value $\rho=3.55 \mathrm{~g} / \mathrm{cm}^{3}$ has been assumed in preliminary tests) it is possible to compute also the corresponding size of the meteoroid.

Fig. 4 shows an example of a meteor track having absolute magnitude $M=+5$ crossing the FOV of JEM-EUSO with a $60^{\circ}$ inclination with respect to the nadir axis. Only a few GTUs have been simulated along the track to solve the memory and data size of the output file issues. At peak intensity, the expected number of counts is around 20

Table 2

Comparison between a typical cosmic ray and a meteor event. The difference in the ratio between the photons at the pupil and those emitted by the two kinds of event takes into account the differences in the distance from the observer and the differences in atmospheric absorption.

\begin{tabular}{lll}
\hline Property & Bright EHECR event & Faint Meteor event \\
\hline Energy/Magnitude & $\mathrm{E}=10^{20} \mathrm{eV}$ & Abs. $\mathrm{M}=+5$ \\
Speed & $3 \times 10^{5} \mathrm{~km} / \mathrm{s}$ & $20 \mathrm{~km} / \mathrm{s}$ \\
Emitted Photons & $10^{16}$ & $10^{18}$ \\
Photons on pupil & $10^{4}$ & $10^{7}$ \\
Duration & $200 \mu \mathrm{s}$ & $0.2 \mathrm{~s}$ \\
\hline
\end{tabular}

per GTU. This confirms the results presented in Table 1, where the detector response was parametrized. The UV night-glow is not simulated, however. As mentioned in Section 2.1, in case of dark nights the background is estimated to be of the order of 1 count/pixel/GTU.

The possibility of simulating flares of a meteor as well as some possible persistent meteor trains has been now implemented in ESAF. Fig. 5 shows an example for both cases. The absolute magnitude of the meteor is $M=+5$ with a duration of $2 \mathrm{~s}$. The flare has a magnitude of $M=+3$ with a duration of $0.5 \mathrm{~s}$, starting $1.5 \mathrm{~s}$ from the beginning of the event. The brightness of the two recorded peaks looks similar because the flare overlaps with the declining brightness of the main meteor signal and, more important, due to the increasing distance of the meteor from the detector in this particular case.

An event including persistent meteor train effects has been also simulated assuming an absolute magnitude $M=+5$. The assumed decay time was $\tau=0.2 \mathrm{~s}$ in this case. We are aware that the assumed brightness of the simulated meteor in this test case is exceedingly faint, to be realistic. The persistence of the meteor train, which is not very evident in this case due to the relative faintness of the simulated event, will be essential to derive the $3 \mathrm{D}$ trajectory and velocity of bright meteors, by exploiting the motion of the ISS.

The Mini-EUSO configuration has also been implemented in ESAF. Due to its much coarser pixel resolution (see Table 3) Mini-EUSO will detect the light-curve with lower signal and spatial resolution. Fig. 6 shows an example of light signal and track detected by Mini-EUSO in case of a meteor with similar zenith angle and magnitude of the one shown in Fig. 4 for JEM-EUSO.

\section{EUSO-TA}

First actual observations of meteor events have been obtained from the ground using EUSO-TA. This is a complete prototype of the JEMEUSO space telescope in operation since 2013 at the Telescope Array (TA) site in Black Rock Mesa, Utah, USA (Kawai et al., 2008). The telescope can perform observations of ultraviolet light generated by cosmic-ray air showers and artificial sources. The aim of the project is to test the JEM-EUSO technology and to study the detector response in conjunction with the TA fluorescence detector.

The telescope is housed in a shed located in front of one of the fluorescence detectors of the Telescope Array collaboration (see Figs. 7a and $b$ ). The detector consists of two $1 \mathrm{~m}^{2}$ sized square Fresnel lenses (see Fig. 7b) and the focal surface consists of one PDM equipped with $6 \times 6$ MAPMTs of $8 \times 8$ pixels (see Fig. 7c) with a field of view of $11^{\circ} \times$ $11^{\circ}$ (FOV of $\sim 0.2^{\circ}$ per pixel). The EUSO-TA apparatus is triggered externally by the TA experiment and images cosmic ray tracks. Fig. 7d shows a typical example. The signal lasts only 1 GTU, as the cosmic ray shower is detected very close to the apparatus (less than $3 \mathrm{~km}$ ). For this reason EUSO-TA can only record packets of 128 GTUs (for a total of $320 \mu \mathrm{s}$ ) of data with a maximum data rate of $20 \mathrm{~Hz}$. In reality, as the typical trigger rate of TA is $\sim 3 \mathrm{~Hz}$, only a few snapshots of any meteor track can be detected. In spite of this difficulty, so far 5 meteor events have been detected. Fig. 8 shows an example of such events. Plot (a) in this figure shows the integrated number of counts in each pixel. A $3 \sigma$ threshold has been applied on each pixel to exclude the signal not due to the meteor. Plots from (b) to (e) show the total number of counts of the meteor track detected by boxes of $5 \times 5$ pixels in the FOV in four consecutive packets. The discontinuity of the signal between packets is due to the discontinuity in which data are acquired. The time difference between the first and the last packet is about $1 \mathrm{~s}$. The measurement is affected by several uncertainties, mainly due to the fact that part of the flux can be lost over non-sensible regions of the detector (zones between different PMTs). We can roughly estimate the magnitude of the meteor at maximum brightness, by computing differences of flux with respect to four different stars in the FOV. Taking into account all the uncertainties, including those related to the conversion of the star magnitudes, given in B colour, we can conclude that this meteor 
a) $3 d$ meteor track

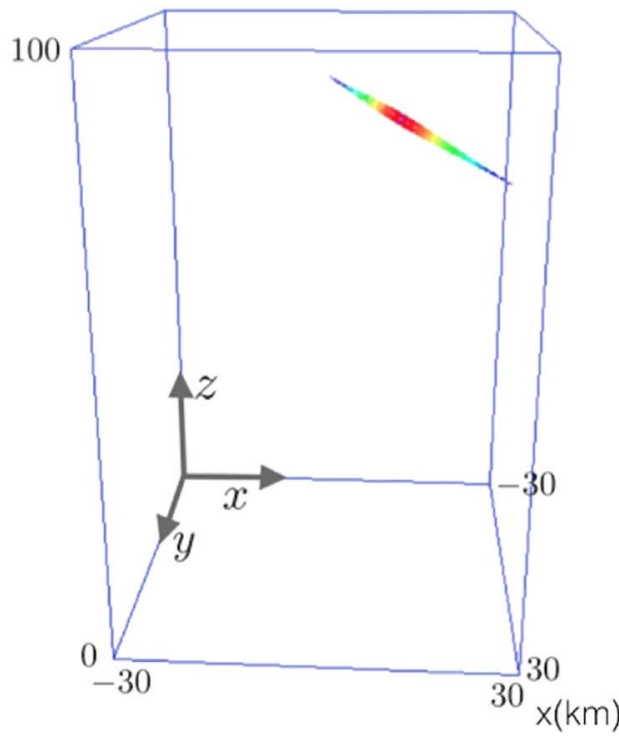

light profile on focal surface

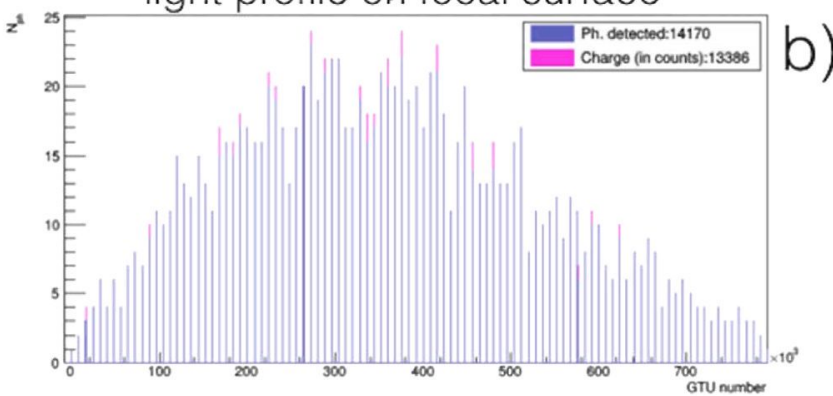

C)

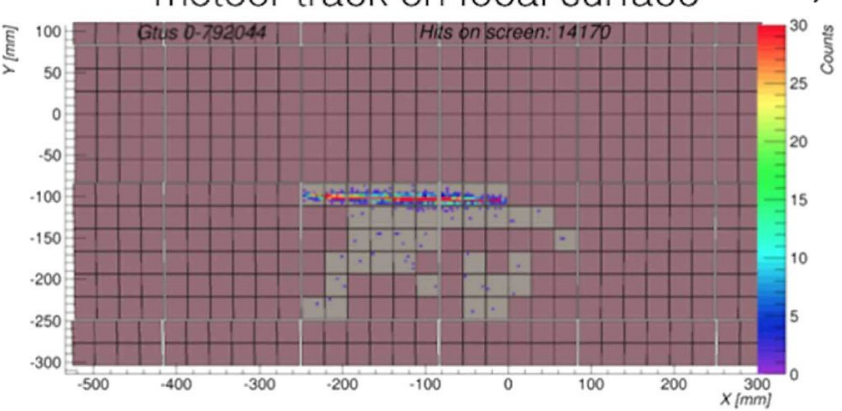

Fig. 4. Example of a meteor track of absolute magnitude $M=+5$ simulated with ESAF. (a): the 3D trajectory of the meteor in the atmosphere. The zenith angle is $60^{\circ}$ from nadir axis. (b): the expected light profile on the detector. Only a few GTUs are simulated. (c): the resulting meteor track on the focal surface.

reached a magnitude of about +2.5 .

Despite the limited amount of information collected for these events, the above figure shows an experimental example on how meteor events would be imaged. An upgrade of EUSO-TA electronics and data acquisition is in progress, by including also a meteor trigger to follow continuously the time evolution of the meteor signal in the FOV of EUSO-TA.

\section{Conclusions}

The analysis presented in Adams et al. (2015e) already showed that JEM-EUSO has the capability to observe meteors down to magnitude $M=+7$. This result is supported by more detailed simulations performed by using the ESAF simulation code. Taking advantage of its large field of view and high detection rate, JEM-EUSO is able to record a statistically significant flux of meteors, both for sporadic and shower meteors. Unaffected by adverse weather conditions, which limit the effectiveness of ground-based meteor observation networks, JEM-EUSO
Table 3

Comparison between JEM-EUSO, Mini-EUSO and EUSO-TA instrument parameters.

\begin{tabular}{llll}
\hline Property & JEM-EUSO & Mini-EUSO & EUSO-TA \\
\hline Pixel number & $3.2 \times 10^{5}$ & 2304 & 2304 \\
FOV at ground $(\mathrm{km})$ & 230 & 150 & $\mathrm{~N} / \mathrm{A}$ \\
Full FOV & $60^{\circ}$ & $40^{\circ}$ & $11^{\circ}$ \\
Lenses diameter $(\mathrm{m})$ & 2.5 & 0.25 & 1.0 \\
Pixel FOV & $0.075^{\circ}$ & $0.8^{\circ}$ & $0.2^{\circ}$ \\
\hline
\end{tabular}

will become a very important facility in the field of meteor studies. A particularly important role can be played in the detection of bright meteors and fireballs, as these events can be detected even during periods of very high sky background. Therefore, monitoring of these events can always be active, whereas the detection of faint meteors requires more optimal observing conditions.

An exciting development, though limited to sufficiently bright a)

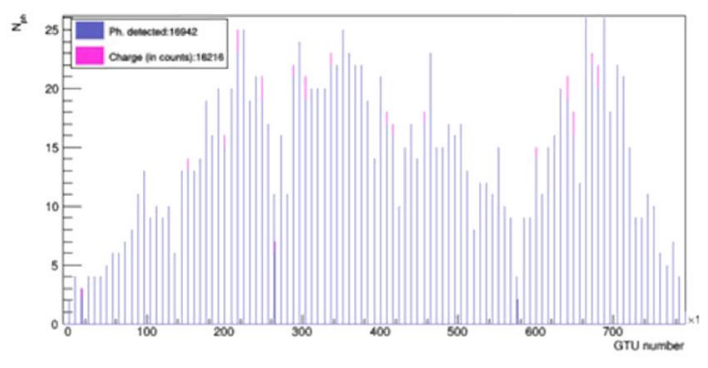

b)

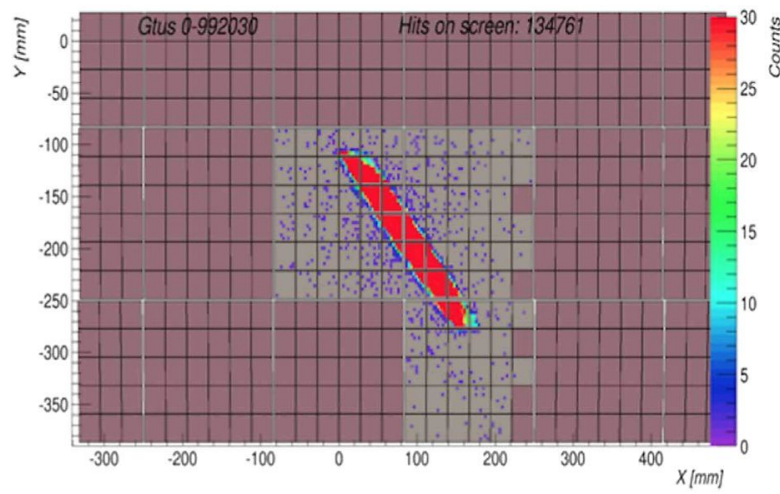

Fig. 5. (a) Expected light profile of a meteor of absolute magnitude $M=+5$ with the presence of a $M=+3$ flare. (b) Meteor signal produced on the focal surface by an event of magnitude $M=+5$ with a persistent meteor train. 
a)

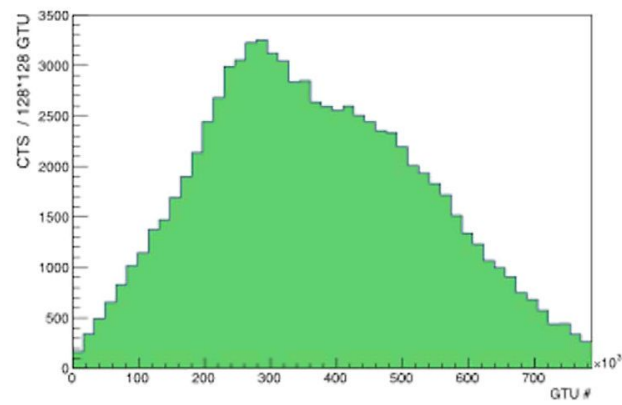

b)

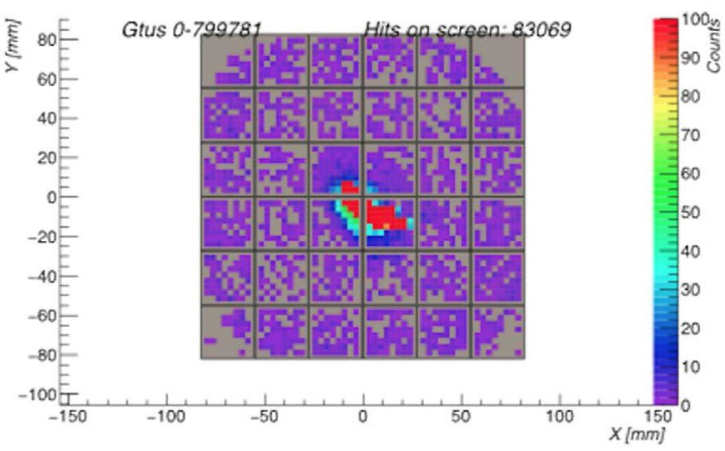

Fig. 6. (a) Expected light profile of a meteor of absolute magnitude $M=+5$ detected by Mini-EUSO. Each time bin on the $x$-axis correspond to 40.96 ms integration time. (b) Expected track on the focal surface (no UV night glow light has been added yet).

b)

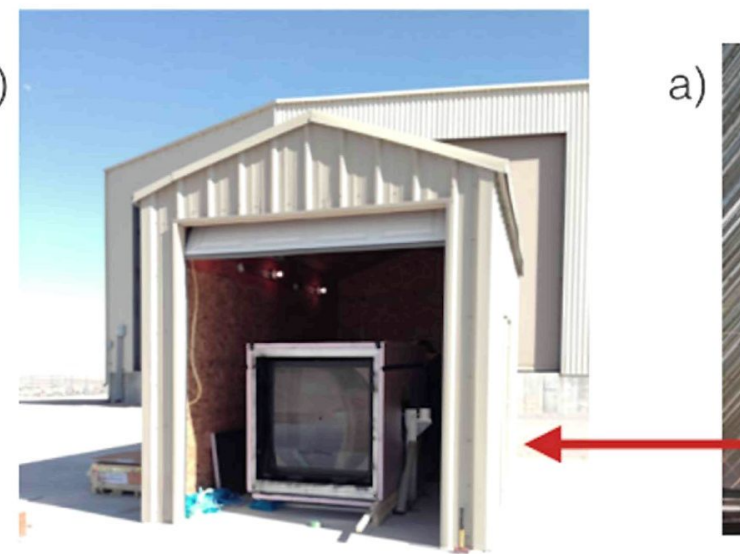

c)

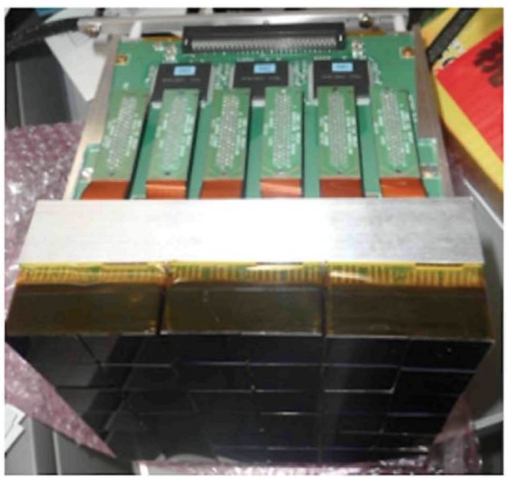

a)

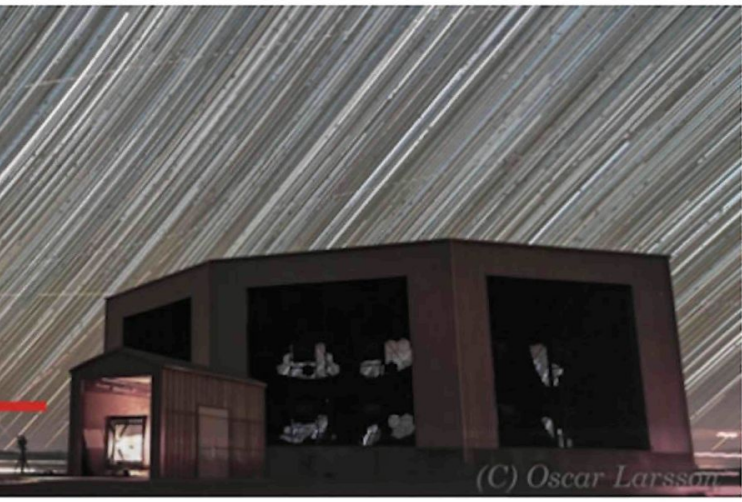

d)

$$
\text { GTU: 284114, pkt 2219, GTU in pket 82, UTC ime: 2015-05-13 06:26:53.3762424, }
$$

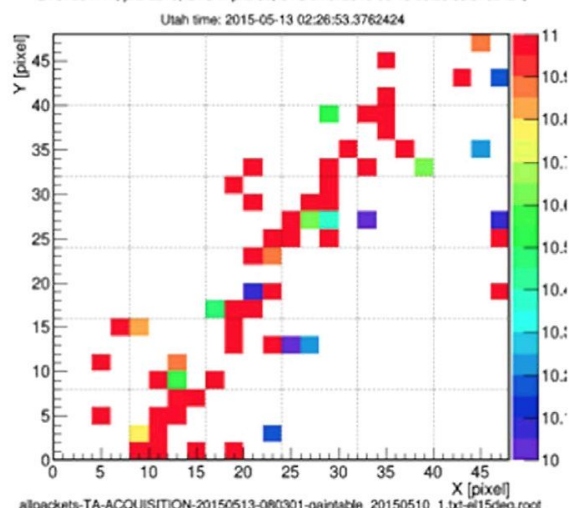

Fig. 7. (a) The TA fluorescence telescope at Black Rock Mesa with the EUSO-TA telescope in front. (b) View of the EUSO-TA telescope with the front Fresnel lens. (c) The PDM of the EUSO-TA telescope. (d) Example of a cosmic ray track of energy $\mathrm{E} \sim 10^{18} \mathrm{eV}$ detected by EUSO-TA.

meteors, is the possibility to exploit the persistence of the meteor train and the movement of the ISS to obtain a 3D reconstruction of the meteor trajectory and velocity, making it possible to compute the original heliocentric orbit of the meteoroid, a very important result for solar system studies.

Our preliminary analysis concerning the possible detection of nuclearites indicates that JEM-EUSO will be sensitive to nuclearites having masses higher than a few $10^{22} \mathrm{GeV} / \mathrm{c}^{2}$. In addition, after a JEMEUSO data acquisition time of only $24 \mathrm{~h}$, it will be able to provide limits on the possible nuclearite flux one order of magnitude lower than the limits reached by the experiments carried out so far.

Prior to JEM-EUSO, the Mini-EUSO pilot mission is expected to fly aboard the ISS on 2017-2018. It will prove the JEM-EUSO observa- tional principle and by means of detailed offline data analysis it will allow us to detect meteors having magnitude as faint as absolute magnitude $M<+5$. Mini-EUSO will be sensitive to nuclearites with mass higher than a few $10^{24} \mathrm{GeV} / \mathrm{c}^{2}$. After one month of operations it could reach a sensitivity of about 2 orders of magnitude better than what has been obtained so far by means of ground experiments.

A ground-based pathfinder mission of JEM-EUSO, named EUSO-TA, is in operation since 2013 in Utah. Despite the fact that the detector is specifically designed to be sensitive to cosmic ray events, a few meteors have been observed, proving on ground the observational principle of JEM-EUSO. There are plans to equip in near future EUSO-TA electronics with a meteor trigger in order to observe more efficiently this category of events. 


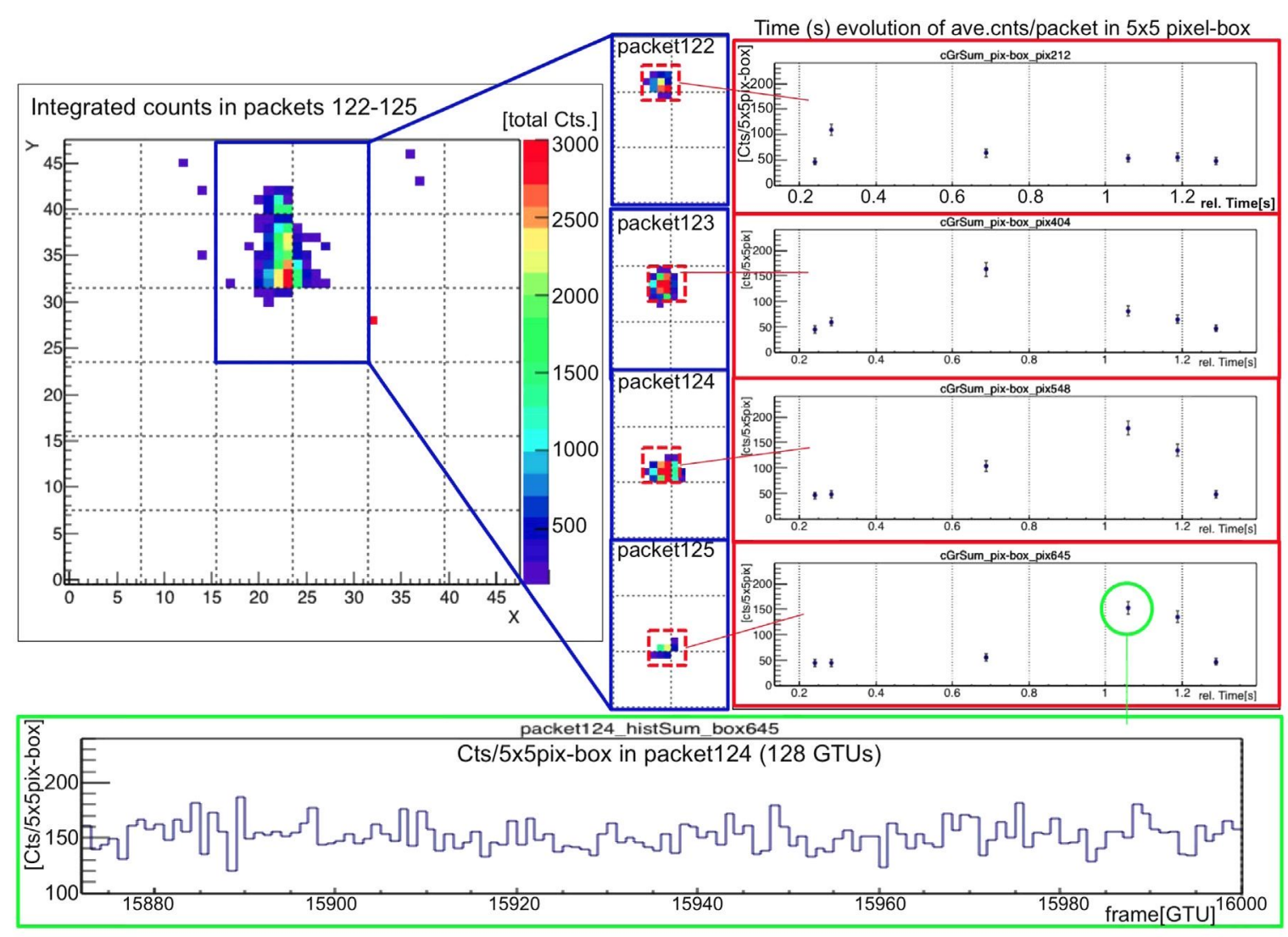

Fig. 8. Meteor track detected by EUSO-TA. The integrated counts corresponding to the whole event are shown in the left. The panels on the right show the light profile of four different blocks of $5 \times 5$ pixels. The morphology of the event shows a fast initial brightening of about 0.9 mag, followed by a slightly slower decline of about 1.7 mag. The panel on the bottom shows for a specific measurement, indicated by a circle in the plot above, the GTU by GTU evolution of the signal.

\section{Acknowledgements}

We thank two anonymous Referees for their detailed and exhaustive reviews, which led to a substantial improvement of this work. We also thank Dr. P. Jenniskens for his kind help in assessing the predictable intensity of meteor light observed at UV wavelengths. This work was partially supported by Basic Science Interdisciplinary Research Projects of RIKEN and JSPS KAKENHI Grant (22340063, 23340081, and 24244042), by the Italian Ministry of Foreign Affairs and International Cooperation, by the 'Helmholtz Alliance for Astroparticle Physics HAP' funded by the Initiative and Networking Fund of the Helmholtz Association, Germany, and by Slovak Academy of Sciences MVTS JEM-EUSO as well as VEGA grant agency project 2/ $0076 / 13$. Russia is supported by the Russian Foundation for Basic Research Grant No 13-02-12175-ofi-m. The Spanish Consortium involved in the JEM-EUSO Space Mission is funded by MICINN \& MINECO under the Space Program projects: AYA2009-06037-E/AYA, AYA-ESP2010-19082, AYA-ESP2011-29489-C03, AYA-ESP201239115-C03, AYA-ESP2013-47816-C4, MINECO/FEDER-UNAH13-4E2741, CSD2009-00064 (Consolider MULTIDARK) and by Comunidad de Madrid (CAM) under projects S2009/ESP-1496 \& S2013/ICE-2822.

\section{References}

Adams Jr., J.H., et al., JEM-EUSO Collaboration, 2015. Exp. Astron. 40, 3-17. Adams Jr., J.H., et al., JEM-EUSO Collaboration, 2015. Exp. Astron. 40, 19-44. Adams Jr., J.H., et al., JEM-EUSO Collaboration, 2015. Exp. Astron. 40, 301-314. Adams Jr., J.H., et al., JEM-EUSO Collaboration, 2015. Exp. Astron. 40, 239-251. Adams Jr., J.H., et al., JEM-EUSO Collaboration, 2015. Exp. Astron. 40, 253-279. Adams Jr., J.H., et al., JEM-EUSO Collaboration, 2013. Astropart. Phys. 44, 76-90. Ambrosio, M., et al., MACRO Coll., 2000. Eur. Phys. J. C 13, 453.

Berat, C., et al., 2010. Astropart. Phys. 33, 221-247.

Bertaina, M., et al., JEM-EUSO Coll., 2014. Adv. Space Res. 53, 1515-1535.

Cecchini, S., et al., SLIM Coll., 2008. Eur. Phys. J. C 57, 525.

De Rujula, A.. Glashow, S.L., 1984. Nature 312, 734-737.

Jacchia, L.G., et al., 1967. Smithson. Contr. Astrophys. 25.

Jenniskens, P., et al., 2002. Meteorit. Planet. Sci. 37, 1071-1078.

Kasuga, T., et al., 2005. A \& A 435, 341-351.

Kawai, H., et al., TA Coll., 2008. Nucl. Phys. B (Proc. Suppl.) 175-176. 221-226.

Nardelli, A., 2014. Meteor simulation in ESAF for the JEM-EUSO mission. Bachelor thesis, università telematica internationale uninettuno.

Pavalas, G.E., et al., 2012. (ANTARES Coll.), In: Proceedings of the 23rd European Cosmic Ray Symposium, Moscow, 543.

Pecina, P.G., Koten, P., 2009. A\& A 499, 313-320.

Price, P.B., 1988. Phys. Rev. D 38, 3813-3814.

Ricci, M., et al., JEM-EUSO Collaboration, 2015. PoS.(ICRC2015) 599.

Robertson, J.B., Ayers, W.G., 1968. NASA Technical Note D-4312; 〈http://ntrs.nasa.gov/ archive/nasa/casi.ntrs.nasa.gov/19680007281.pdf >

Shrbený, L., Spurný, P., 2009. A 506, 1445-1454. 